\title{
Radical Tunes
}

\section{Exploring the Impact of Music on Memorization of Stroke Order in Logographic Writing Systems}

\author{
Oleksandra Keehl \\ University of California, Santa Cruz \\ Santa Cruz, CA \\ okeehl@ucsc.edu
}

\author{
Edward Melcer \\ University of California, Santa Cruz \\ Santa Cruz, CA \\ emelcer@ucsc.edu
}

\begin{abstract}
Music and rhythm are powerful tools that can be employed to enhance learning and memory. While games are commonly utilized to aid in second language acquisition, few have explored the implications of sound on learner's ability to draw and remember logographic characters (such as those in Chinese hanzi, Japanese kanji and Korean hanja). We created Radical Tunes, a kanji drawing music game, to explore the impacts of incorporating music on players' ability to retain meaning and stoke order of several kanji. In this paper, we describe the design rationale for Radical Tunes, and present results from a pilot study comparing a music focused version of the game with one that uses non-musical sound effects. Results show that while both conditions improved players' shortterm ability to remember/draw kanji, there were no significant differences in improvement between the conditions. However, the use of music did improve immersion-an important factor related to learning. This work has implications for designers of second language acquisition games, and how they can incorporate rhythm and music into their games to increase player engagement.
\end{abstract}

\section{KEYWORDS}

Educational Game; Music; Immersion; Second-Language Acquisition; Kanji; Radicals

ACM Reference Format:

Oleksandra Keehl and Edward Melcer. 2019. Radical Tunes: Exploring the Impact of Music on Memorization of Stroke Order in Logographic Writing Systems. In The Fourteenth International Conference on the Foundations of Digital Games (FDG '19), August 26-30, 2019, San Luis Obispo, CA, USA. ACM, New York, NY, USA, 6 pages. https://doi.org/10.1145/3337722.3337764

\section{INTRODUCTION}

Logographic writing systems, such as Chinese, Japanese and Korean, are notoriously difficult to learn. The students of these languages have to copy the characters dozens of times in order to memorize them. As a result, acquiring hundreds of characters through rote learning is a daunting task and can discourage a casual learner from pursuing a language.

Permission to make digital or hard copies of all or part of this work for personal or classroom use is granted without fee provided that copies are not made or distributed for profit or commercial advantage and that copies bear this notice and the full citation on the first page. Copyrights for components of this work owned by others than ACM must be honored. Abstracting with credit is permitted. To copy otherwise, or republish, to post on servers or to redistribute to lists, requires prior specific permission and/or a fee. Request permissions from permissions@acm.org.

FDG '19, August 26-30, 2019, San Luis Obispo, CA, USA

(c) 2019 Association for Computing Machinery.

ACM ISBN 978-1-4503-7217-6/19/08 . \$ \$15.00

https://doi.org/10.1145/3337722.3337764
Games have become a frequently utilized tool to aid in secondlanguage acquisition (SLA), addressing topics such as vocabulary and meaning [7, 21], tones [11], conversational phrases [5], and culture [2]. However, there has been relatively little focus on how SLA games can be employed to teach writing skills for logographic characters. For instance, while there are many educational games and applications out there intended to help people memorize Japanese kanji, most focus solely on the reading and meaning portions and don't teach stroke order (such as the popular service WaniKani ${ }^{1}$ ). However, the stroke order is also important to learn, as it is instrumental for reading and writing handwritten scripts. Furthermore, the majority of commercial writing apps primarily amount to digital flashcards. While convenient and useful to a motivated learner, they offer little extrinsic motivation to a casual student, which is a significant hurdle for many kanji students [18].

To address these issues, we created a musical game for learning to write kanji. The name of our game, Radical Tunes, is not incidental: radicals are the building components of kanji (Figure 1), and music can play an important role in memory and learning. For instance, in a study by Wallace [22], subjects were best able to memorize lyrics to a song if it was played to them several times with the same rhythmically matching melody (as opposed to several matching melodies or repeating but mismatched melody). Our hope is that the musical aspects of the game will turn the tedious task of repeatedly drawing the kanji into an enjoyable and educationally-effective activity. To achieve this we are relying on the power of music as a mnemonic device [22] and popularity of rhythm games, such as $\mathrm{Osu}^{2}$ which has over 13 million registered players.

Beyond its aesthetic appeal and mnemonic potential, music has also been found to play a notable role in altering immersion $[14,20]$. Cairns et al. [1] describe immersion as "the degree of involvement that players have with different aspects of the game leading to a move of the attention, awareness and thoughts of the player from the real world around them to the events happening within the game". This suggests immersion is an important component of the gaming experience, and other research has shown that it can also enhance learning in a number of ways $[3,6]$, making it important to both SLA games and educational games in general.

In this paper, we provide the design rationale for Radical Tuneswhich ties unique melodies to radicals within a kanji. We also present results from a pilot study exploring whether adding music to an SLA game would improve learning outcomes and key factors that can impact learning, such as immersion. To our knowledge, no prior work has examined the relationship between music and

\footnotetext{
${ }^{1}$ https://www.wanikani.com

${ }^{2}$ https://osu.ppy.sh/home
} 

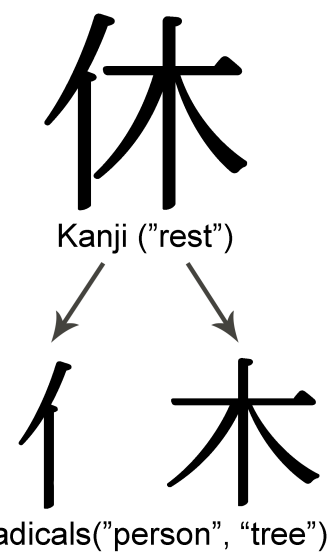

Radicals("person", "tree")
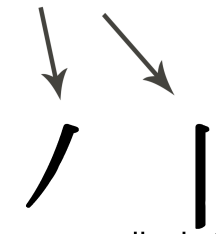

Person radical strokes

Figure 1: Anatomy of kanji. Each kanji consists of one or more radicals and each radical has one or more strokes.

efficacy of an SLA game for learning the writing stroke order of kanji characters.

\section{RELATED WORK}

The following subsections provide a brief overview of games created to teach various aspects of a language, and notable prior research examining the relationship of music, immersion, and learning.

\subsection{Second-Language Acquisition Games}

Many studies have been conducted on the use of games in SLA, including exploration of kinesthetic learning [8,23], augmented reality [16], alternate reality [4], collaborative learning [4, 9, 11, $16,21,23]$, and roleplaying [2] in games. These games addressed various aspects of language acquisition, such as vocabulary and meaning $[2,7,15]$, pronunciation and tones [9, 11, 17], grammar [4], writing [21, 23] and even culture $[2,16]$.

Notably, many of these studies showed that one of the main benefits from including games in SLA was increased engagement. Multiple findings highlighted that the majority of players felt entertained and challenged, which could lead to dedicating more time to the learning activity. Other benefits observed in some of the games were ease of access (on mobile devices) [11] and reduced stress (from not having to perform in front of teachers/peers) [4] However, to our knowledge, no one has conducted a study on the effects of music on memorization of kanji or other logographic characters.

In addition to positive outcomes of games on learning language, some prior work has found negative effects as well. Specifically, DeHaan et al. has studied effects of cognitive load in interactive

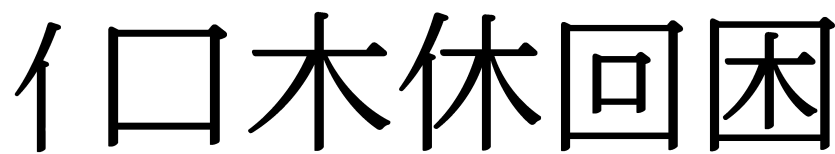

Figure 2: Radicals and kanji used in study. Left to right: person, mouth, tree, rest, episode, annoyed.

games on learning [7]. Using a commercial musical vocabulary learning game Parappa the Rapper 2, they compared vocab retention from students who played the game and those who watched the gameplay without any interaction. In their study, the watching students retained more vocabulary than the players, which lead the authors to caution that it is possible for interactivity in a game to affect learning outcomes negatively. We kept this concern in mind when designing Radical Tunes. In Parappa the Rapper 2, the player had to hit sequences of buttons in time with music. One could master this activity without paying any attention to the words. In Radical Tunes, the music is created as the player draws each stroke, and thus the game's interactivity is integral to the learning activity instead of distracting from it.

\subsection{Immersion, Music, and Learning}

Music has been linked to various aspects of human performance. One study suggested that calming music helped increase children's performance in math, word memorization and altruistic tendencies [10]. Another study examined effects of music on lyrics memorization and found that subjects exposed to a repeating melody with a rhythm matching the lyrics performed better than those listening to a mismatched, altering or no melody[22].

Music has also been linked to immersion in digital games [20], and immersion, in turn, has been shown to have a positive effect on learning [2,3]. Many of the papers mentioned in the SLA section, while not explicitly mentioning immersion, listed engagement as a factor leading to positive learning outcomes. Notably, engagement is recognized as one of the core components of immersion [1,3]. Immersion is therefore another important factor to examine in our study.

\section{RADICAL TUNES}

Radical Tunes is a musical game for learning to write kanji, the Japanese characters borrowed from Chinese language. Kanji consist of radicals and strokes (Figure 1). Radicals are building blocks of kanji, and each kanji has at least one radical. Each radical has a meaning, which may or may not contribute to the meaning of the kanji, and these are often used in mnemonics. WaniKani ${ }^{3}$ uses made-up humorous meanings for radicals, which often have little to do with the original meaning, but through the shock value have a strong mnemonic effect. Each radical consists of one or more strokes. Strokes don't carry a meaning on their own.

Many mnemonic devices exist for remembering the shape and meaning of a kanji [19], but to our knowledge, none of these are focused on learning the proper writing stroke order-which is necessary for handwriting proficiency. As a result, we created Radical

\footnotetext{
${ }^{3}$ https://www.wanikani.com
} 

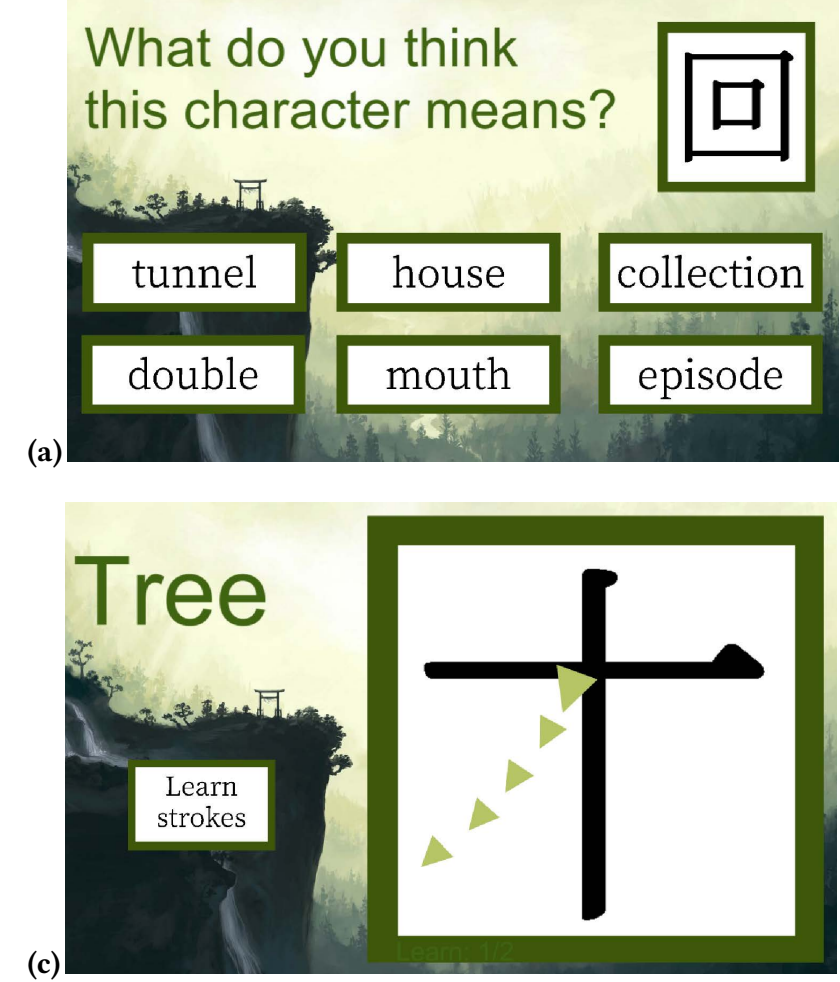
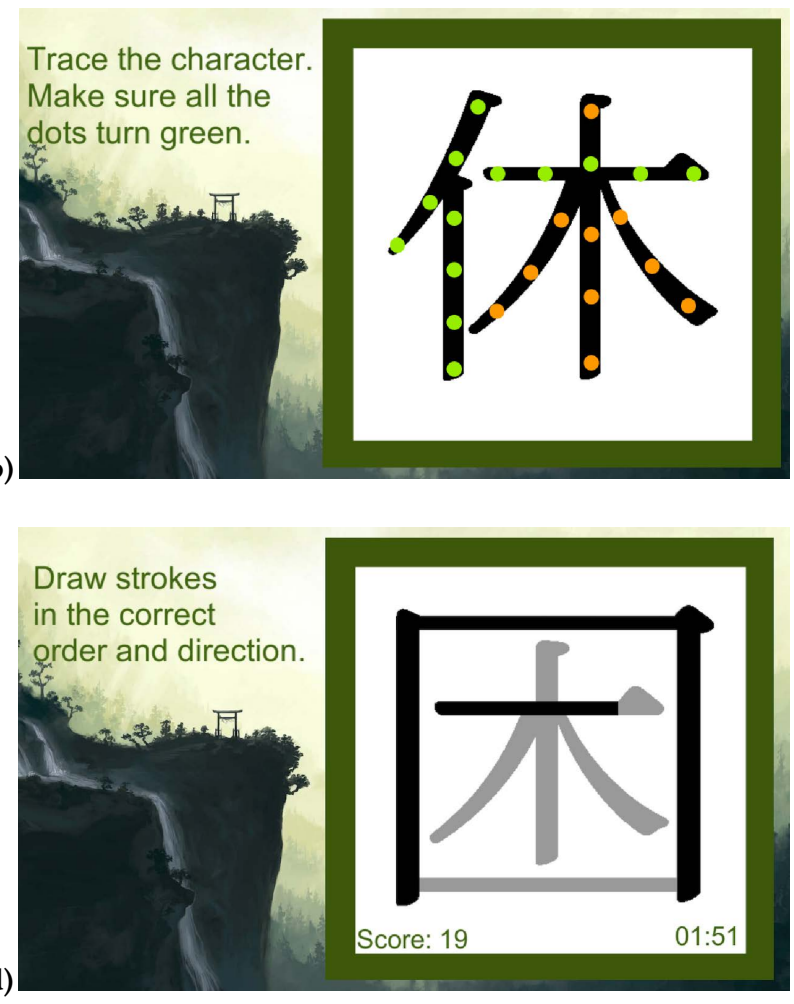

Figure 3: Radical Tunes screenshots. (a) Meaning pre- and post-test. (b) Stroke order pre- and post-test. (c) Stroke learning screen. (d) Timed and scored game portion.

Tunes to fill that void. Past studies have shown the positive effects of music on memorization of text, especially when it is rhythmically aligned with the material [22]. We wanted to explore if this music mnemonic effect could be extended to memorization of the stroke order of a kanji.

Radical Tunes achieves the intended musical mnemonic mechanism by employing a unique instrument and melody for each radical, thus giving each radical a melodic fingerprint of sorts. These melodies are further broken down, with each element of the melody corresponding to a stroke within the radical. As a result, as the student correctly draws a kanji, they will hear a unique melody corresponding to that kanji unfolding with every stroke.

For our initial game we selected three of the six most commonly occurring radicals in the Jōyo kanji (the 2,136 characters required for baseline literacy). We also selected three kanji which consisted of combinations of those radicals, to add increased complexity, for a total of six characters for our subjects to learn (Figure 2).

Radical Tunes teaches writing stroke order in three stages. First, an animated demo of the stroke order is shown. Next, a player is asked to trace the character with the help of arrows (Figure 3c), and finally, they are encouraged to practice tracing the character without the arrow order hints. If they draw a stroke incorrectly, a soft ping alerts them of the mistake and they will have to attempt the stroke again. After learning is complete, the player can play the game, where they have to draw the kanji correctly and as quickly as possible to earn the most points (Figure $3 \mathrm{~d}$ ).

\section{METHODS}

The goal of our pilot study was to investigate the impact that music and rhythm would have on learning in Radical Tunes. Given that immersion can play an important role in learning for educational games [3, 6] and music has been shown to impact immersion [20], we also wanted to examine if the musical nature of Radical Tunes increased player immersion.

Our study had two versions of the game: 1) the original Radical Tunes with unique tunes for each radical and melodic elements accompanying each stroke; and 2) the control version where the music was replaced with the sounds of chalk writing on a blackboard.

\subsection{Procedure}

The participants were told that the study was for an educational game where they would learn the meaning and writing of six Japanese characters. They were then randomly assigned to either the music or control condition and given an Android tablet with the appropriate version of Radical Tunes loaded. For the experiment, meaning and stroke order tests were built into the beginning and end of the game (Figures $3 a$ and $3 b$ ).

For the meaning tests, the participants were shown the characters one at a time and had to chose an answer from 6 possible meanings 
Table 1: IEQ mean scores, standard deviations, significant differences between the two conditions, and the effect size, which is in the medium to large range for significant differences.

\begin{tabular}{lcccccc}
\hline & \multicolumn{2}{c}{ Control } & \multicolumn{2}{c}{ Music } & Significance & Effect Size \\
IEQ Measures & $\mu$ & $\sigma$ & $\mu$ & $\sigma$ & $p$ & $r$ \\
\hline IEQ Overall & 100.33 & 18.1 & 115.25 & 15.29 & $\mathbf{. 0 4}^{*}$ & .41 \\
Cognitive Involvement & 30.08 & 7.01 & 36.08 & 5.13 & $\mathbf{. 0 2 6}^{*}$ & .44 \\
Real World Dissociation & 25.58 & 6.49 & 28.5 & 5.81 & .26 & .23 \\
Emotional Involvement & 12.33 & 3.58 & 15.58 & 3.48 & $\mathbf{. 0 3 4}^{*}$ & .42 \\
Challenge & 16.33 & 1.5 & 17.67 & 1.83 & .063 & .37 \\
Control & 16 & 3.54 & 17.42 & 3.15 & .31 & .21 \\
\hline
\end{tabular}

(Figure 3a). The options were the same for pre- and post-tests. At the end, the players were notified how many characters they identified correctly, though not which ones. This was done to prevent the participants from learning character meanings during the pretest via confirmation of their guesses.

For the stroke order tests, the participants were asked to trace each character on the screen with their finger. We placed orange dots along the lines, and the dots turned green when touched, to indicate which sections of a kanji were already traced (Figure 3b) No feedback was given on this evaluation, as we were examining their knowledge of stroke order without any hints.

After the pre-tests for meaning and stroke order, the participants were taken through the educational section of Radical Tunes. First, the subjects were shown the animation of the correct stroke order with accompanying sounds. Then, they were asked to draw the strokes by following the arrows on the screen (Figure 3c). Finally, they were asked to trace the character unassisted. A soft ping would let them know if they made a mistake, and they would have to redraw the latest stroke. To ensure that each participant received the same amount of instruction, we limited each step to two unskippable repetitions.

Next, participants played the Radical Tunes game by tracing the characters appearing on the screen as quickly and correctly as possible (Figure $3 \mathrm{~d}$ ). The game included each character twice in a randomized order. The same randomized order was used for all participants.

After the game was completed, the final score and time were displayed and the participants were directed to take the meaning and stroke order post-tests. Lastly, they completed a demographic and experience questionnaire on a laptop.

\subsection{Participants}

Twenty four participants took part in the study. They were recruited through class announcements and by word of mouth outside of the university. There were 11 female, 12 male and one non-binary participant. The ages ranged from 19 to 51 with the average age of 25.6 (SD = 7.8). During the study, participants were randomly assigned to one of the two conditions: music ( 7 female, 5 male) and control (4 female, 1 non-binary, 7 male). All participants reported no prior knowledge of Japanese or Chinese.

\subsection{Measures}

4.3.1 Immersive Experience Questionnaire (IEQ). We used the Immersive Experience Questionnaire [13] to measure the level of immersion experienced by our subjects. IEQ takes into account five dimensions of immersion: cognitive involvement, emotional involvement, real world dissociation, control and challenge. The cognitive involvement dimension covers factors like attention and effort the subjects invested into the game. Emotional involvement covers the degree to which the subjects enjoyed the game and were interested in seeing more of it. The real world dissociation measures the degree to which the participants lost track of time and their surroundings. The control dimension assesses the perceived responsiveness and ease of use of controls. The challenge dimension measures the perceived difficulty of the game.

In their paper, Jennett et al. suggested that immersion is an important component of what is perceived as a good game. For Radical Tunes to serve its educational purpose, it is important for it to be able to hold the players' attention and provide an enjoyable experience that entices them to return. With this in mind, we decided that IEQ would help us measure how well Radical Tunes performed in this sense.

4.3.2 Radical Tunes scores. The participants were given pre- and post-tests for meaning (Figure 3a) and stroke order (Figure 3b) for each of the six characters. We used the relative improvement between the pre/post tests to assess the learning outcome of the game.

\section{RESULTS}

\subsection{Prior Knowledge and Experience}

According to a series of independent samples t-tests, participants in the two conditions did not differ with respect to prior video game experience $(\mathrm{p}=.61)$, prior rhythm game experience $(\mathrm{p}=.81)$, and on the pre-test learning outcome measures: kanji meaning $(\mathrm{p}=.82)$ and stroke order $(\mathrm{p}=.31)$.

We can therefore assume that participants in both groups had similar prior game experience and knowledge of kanji for the following analyses.

\subsection{Immersion Experience}

We first examine participants' experience of immersion during gameplay for both conditions (Table 1). To analyze differences between IEQ scores for the two conditions we used an independent 
Table 2: Descriptive statistics of learning outcomes (meaning tests, stroke order tests, and in-game scores).

\begin{tabular}{lcccc}
\hline & \multicolumn{2}{c}{ Control } & \multicolumn{2}{c}{ Music } \\
Learning Outcome Measures & $\mu$ & $\sigma$ & $\mu$ & $\sigma$ \\
\hline Meaning Pre-test & 1.08 & .9 & 1.16 & .84 \\
Meaning Post-test & 5.5 & 1 & 5.58 & .67 \\
Meaning Improvement & +4.42 & 1.73 & +4.42 & 1.17 \\
\hline Stroke Order Pre-test & .33 & .65 & .59 & .52 \\
Stroke Order Post-test & 5.41 & .9 & 5.67 & 1.16 \\
Stroke Order Improvement & +5.08 & .9 & +5.08 & 1.38 \\
\hline In-Game Score & 102.17 & 8.93 & 106.58 & 2.19 \\
\hline
\end{tabular}

samples t-test. Table 1 shows descriptive statistics for IEQ scores, as well as significant differences and effect sizes between conditions. Results found a significant difference in favor of the music condition of the game increasing overall IEQ scores $(\mathrm{p}=.04, \mathrm{r}=.41)$, cognitive involvement $(\mathrm{p}=.026, \mathrm{r}=.44)$, and emotional involvement $(\mathrm{p}=.034$, $\mathrm{r}=.42$ ). There were no significant differences for real world dissociation, challenge, or control (all p's $>.063$ ). This suggests that adding melodies and music to our game helped to improve player factors (cognitive involvement and emotional involvement) but not game factors (challenge and control) of immersion [13].

\subsection{Learning Outcomes}

To better understand players' learning outcomes from the game we analyzed pre- and post-test scores for meaning and stroke order, as well as final scores from the game portion of Radical Tunes. Descriptive statistics for the three measures are shown in Table 2. A series of independent samples t-tests showed that both conditions had a significant increase in both meaning and stroke order scores from pre- to post-test: control (meaning - $\mathrm{p}<.001, \mathrm{r}=.95$; stroke order $-\mathrm{p}<.001, \mathrm{r}=.96$ ), and music (meaning $-\mathrm{p}<.001, \mathrm{r}=.92$; stroke order $-\mathrm{p}<.001, \mathrm{r}=.94$ ). This suggests that the game itself was indeed successful for short-term teaching of kanji meanings and writing stroke orders to players. However, there were no significant differences in improvement between conditions for meaning, stroke order, or in-game score (all p's > .11).

\subsection{Perception of Usefulness}

We asked participants to rate if they would recommend this game to someone trying to learn Japanese or Chinese on a 7-point Likert scale (1 - not at all to 7 - very likely). Scores were notably higher for the music condition $(\mu=6.58, \sigma=.67)$ than the control condition ( $\mu=5.42, \sigma=1.78)$. An independent samples t-test showed that there was a significantly higher likelihood of recommending the game for the music condition ( $\mathrm{p}>.045, \mathrm{r}=.4$ ).

\section{DISCUSSION}

\subsection{Immersion and Learning in an SLA Game}

The music condition participants showed significantly more overall immersion according to IEQ responses (Table 1), particularly in the cognitive and emotional involvement dimensions. This falls in line with existing studies showing increased immersion with addition of pleasing music [20]. We expected challenge and control to be similar across the two groups, since the game controls and tasks were identical. Overall, this indicates that adding music to Radical Tunes-and likely SLA games in general-can improve player factors (cognitive involvement and emotional involvement) but not game factors (challenge and control) of immersion [13].

Both conditions showed a significant improvement between preand post-test scores for meaning and stroke order (all p's < .001)indicating that the game is effective for helping players learn. However, there was no significant difference in the improvement between the two conditions (Table 2). This is possibly due to the limited number of kanji tested and short-term interaction with Radical Tunes, not providing enough time and difficulty for the music mnemonic approach to have a significant impact on learning outcomes.

\subsection{Limitations}

While the immersion and perceived usefulness results are promising, showing that players tend to prefer the music version of Radical Tunes, we weren't able to see any effects of music on memorization of stroke order or kanji meaning. We theorize that these effects may present themselves better over time with a larger number of kanji. Furthermore, the number of participants in our study $(n=24)$ is lower than $n=30$ recommended to reach normal data distributions for our observed effect size (this rule of thumb is derived from the Central Limit Theorem [12]). This limits the scope of our results. To remedy these limitations, we plan to conduct a future longitudinal study with a larger number of participants and with expanded learning content to better observe the effects of kanji-specific melodies on long-term stroke order memorization abilities of students.

\subsection{Future Design Improvements}

Our current implementation of Radical Tunes features melodic elements of a fixed duration in what we estimated would be the appropriate length for each stroke to be drawn. Each element is triggered when the player correctly begins to draw a stroke. If the player goes out of bounds or lifts their finger half way through, the melody is interrupted, which works as designed. However, if the speed of the player's drawing is significantly different from our estimate, the melody and drawing become out of sync. Several of the participants in the music condition of Radical Tunes noted this issue and expressed their wish for a better coupling of melody and stroke speed. Dynamically adjusting stroke sounds is something we intend to implement for our next round of experiments. Some participants were also dissatisfied with the slower pacing of the game, which resulted from us enforcing two repetitions of each of the learning steps. In the next iteration, we plan to allow students to navigate through sections of the game freely and learn things at their own pace.

\section{CONCLUSIONS}

In this paper we described Radical Tunes-a musical game for learning to write Japanese kanji, which uses unique melodies to aid with stroke order memorization. We also described the results of our pilot study, which compared the musical version of Radical Tunes 
with a version which instead had non-musical sound effects, i.e., the sound of chalk writing on a blackboard. Through use of the Immersive Experience Questionnaire, we found that incorporation of music in Radical Tunes significantly increased player immersion. The participants from both conditions showed significant score improvement between pre- and post-tests, which shows that Radical Tunes was effective in teaching the kanji to players, at least in the short-term. The small scope of our pilot study-only six charactersdidn't expose any mnemonic effects the music may have on players' ability to retain the kanji long term, and it is something we plan to investigate further in our future work.

\section{REFERENCES}

[1] Paul Cairns, Anna Cox, and A Imran Nordin. 2014. Immersion in digital games: review of gaming experience research. Handbook of digital games 1 (2014), 767.

[2] Alan Cheng, Lei Yang, and Erik Andersen. 2017. Teaching language and culture with a virtual reality game. In Proceedings of the 2017 CHI Conference on Human Factors in Computing Systems. ACM, 541-549.

[3] M-T Cheng, H-C She, and Leonard A Annetta. 2015. Game immersion experience: its hierarchical structure and impact on game-based science learning. Fournal of Computer Assisted Learning 31, 3 (2015), 232-253.

[4] Thomas M. Connolly, Mark Stansfield, and Thomas Hainey. 2011. An Alternate Reality Game for Language Learning: ARGuing for Multilingual Motivation Comput. Educ. 57, 1 (Aug. 2011), 1389-1415.

[5] Gabriel Culbertson, Shiyu Wang, Malte Jung, and Erik Andersen. 2016. Social situational language learning through an online 3D game. In Proceedings of the 2016 CHI Conference on Human Factors in Computing Systems. ACM, 957-968.

[6] Chris Dede. 2009. Immersive interfaces for engagement and learning. science 323, 5910 (2009), 66-69.

[7] Jonathan deHaan, W. Michael Reed, and Katsuko Kuwada. 2010. The effect of interactivity with a music video game on second language vocabulary recall. Language Learning \& Technology 14, 2 (2010), 74-94.

[8] Darren Edge, Kai-Yin Cheng, and Michael Whitney. 2013. SpatialEase: Learning Language Through Body Motion. In Proceedings of the SIGCHI Conference on Human Factors in Computing Systems (CHI '13). ACM, New York, NY, USA, 4.

[9] Xiangmin Fan, Wencan Luo, and Jingtao Wang. 2017. Mastery Learning of Second Language through Asynchronous Modeling of Native Speakers in a Collaborative Mobile Game. In Proceedings of the 2017 CHI Conference on Human Factors in Computing Systems. ACM, 4887-4898.

[10] Susan Hallam, John Price, and Georgia Katsarou. 2002. The Effects of Background Music on Primary School Pupils' Task Performance. Educational Studies 28, 2 (2002), 111-122.

[11] Andrew Head, Yi Xu, and Jingtao Wang. 2014. Tonewars: Connecting language learners and native speakers through collaborative mobile games. In International Conference on Intelligent Tutoring Systems. Springer, 368-377.

[12] C.C. Heyde. 2006. Central Limit Theorem. In Encyclopedia of Actuarial Science. American Cancer Society.

[13] Charlene Jennett, Anna L. Cox, Paul Cairns, Samira Dhoparee, Andrew Epps, Tim Tijs, and Alison Walton. 2008. Measuring and Defining the Experience of Immersion in Games. Int. f. Hum.-Comput. Stud. 66, 9 (Sept. 2008), 641-661.

[14] Kari Kallinen. 2004. The effects of background music on using a pocket computer in a cafeteria: Immersion, emotional responses, and social richness of medium. In Extended Abstracts on Human Factors in Computing Systems (CHI '04). ACM.

[15] Anuj Kumar, Pooja Reddy, Anuj Tewari, Rajat Agrawal, and Matthew Kam. 2012 Improving literacy in developing countries using speech recognition-supported games on mobile devices. In Proceedings of the SIGCHI Conference on Human Factors in Computing Systems. ACM, 1149-1158.

[16] Zheng Liu, Holden. 2016. Analyzing students' Language Learning Experience in an Augmented Reality Mobile Game: An Exploration of an Emergent Learning Environment. In Procedia - Social and Behavioral Sciences. Elsevier BV, 369-374.

[17] Musa Nushi, Samaneh Shafeie, and Meisam Shafiei. 2017. Game-based Teaching of Stress Placement on Multi-syllabic English Words. Journal of Teaching English Language Studies 5 (01 2017), 46-64.

[18] Heath Rose. 2017. The Japanese writing system: Challenges, strategies and selfregulation for learning kanji. Multilingual Matters.

[19] Michael Rowley. 1992. Kanji pict-o-graphix: over 1,000 Japanese kanji and kana mnemonics. Stone Bridge Press.

[20] Timothy Sanders and Paul Cairns. 2010. Time perception, immersion and music in videogames. In Proceedings of the 24th BCS interaction specialist group conference. British Computer Society, 160-167.

[21] Michael B Syson, Ma Regina E Estuar, and Karl T See. 2012. Abkd: Multimodal mobile language game for collaborative learning of chinese hanzi and japanese kanji characters. In Proceedings of the The 2012 IEEE/WIC/ACM International foint
Conferences on Web Intelligence and Intelligent Agent Technology-Volume 03. IEEE Computer Society, 311-315.

[22] Wanda T. Wallace. 1994. Memory for music: Effect of melody on recall of text. Journal of Experimental Psychology: Learning, Memory, and Cognition 20 (11 1994), 1471-1485.

[23] Kelly Yap, Clement Zheng, Angela Tay, Ching-Chiuan Yen, and Ellen Yi-Luen Do. 2015. Word out!: Learning the Alphabet Through Full Body Interactions. In Proceedings of the 6th Augmented Human International Conference (AH '15). ACM, New York, NY, USA, 101-108. 